\title{
CONTROLE LINEAR DE MÁXIMO TORQUE DE UM MOTOR SÍNCRONO DE ÍMÃS PERMANENTES INTERIORES
}

\author{
Daniel Figueiredo* \\ danielfm@weg. net
}

\author{
Edson Bim $^{\dagger}$ \\ bim@dsce.fee.unicamp.br
}

\author{
${ }^{*}$ Weg Automação \\ Av. Prefeito Waldemar Grubba, nº 3000 \\ Jaraguá do Sul, SC- Brasil \\ ${ }^{\dagger}$ Unicamp, FEEC \\ Rua Albert Einstein, ${ }^{\circ} 400$ \\ Cidade Universitária Zeferino Vaz, Campinas-SP-Brasil
}

\section{RESUMO}

Neste artigo é apresentado o controle linear de torque de um motor síncrono de ímãs permanentes interiores funcionando nas regiões de torque constante e de potência constante. O controle de torque constante é implementado através da curva de máximo torque por Ampère do motor. A tensão de terminal do estator é mantida constante ao longo da região de enfraquecimento mediante o controle da reação de armadura. Para melhorar a resposta dinâmica do controle, o controle de máximo torque por Ampère é estendido à região de enfraquecimento parcial de campo, que é uma região intermediária de velocidade. Resultados de simulação computacional e experimentais são obtidos com o objetivo de validar os métodos de controle propostos.

PALAVRAS-CHAVE: Motor síncrono de ímãs permanentes interiores, controle linear de torque, controle de máximo torque por Ampère e de enfraquecimento de campo.

\begin{abstract}
Linear Torque Control of an Interior Permanent Magnet Synchronous Motor

In this article is presented the linear torque control of an interior permanent magnet synchronous motor operating in the constant torque and field weakening ranges. The constant torque control is implemented by using the maximum torque per Ampère curve of the motor. The terminal stator voltage remains constant along the field weakening range by controlling the armature reaction control. In order to obtain a better dynamic response, the maximum torque per Ampère control is extended up to the field weakening range, called partial field weakening region. Experimental and simulation results are presented in order to validate the proposed torque control methods.
\end{abstract}

KEYWORDS: Interior permanent magnet synchronous motor (IPMSM), linear torque control, maximum torque per Ampère control and field weakening control 


\section{LISTA DE SÍMBOLOS}

$\begin{array}{ll}\alpha, \beta & \text { Coordenadas do sistema estacionário } \\ d, q & \text { Coordenadas do sistema síncrono } \\ \theta_{r} & \text { Angulo do rotor } \\ \omega_{b} & \text { Velocidade de base do motor } \\ \omega_{r} & \text { Velocidade síncrona do rotor } \\ \omega_{P} & \text { Velocidade que separa as regiões de torque } \\ & \text { constante e potência constante } \\ \psi_{m} & \text { Fluxo de magnetização do entreferro } \\ \psi_{a m} & \text { Fluxo da armadura que atravessa o entreferro } \\ \psi_{a M} & \text { Fluxo dos ímãs que concatena a armadura } \\ L_{a M} & \text { Indutância mútua entre a armadura e o rotor } \\ i_{M} & \text { Corrente fictícia dos ímãs } \\ V_{s} & \text { Tensão de terminal da armadura } \\ I_{s} & \text { Corrente de armadura } \\ E_{a M} & \text { Tensão a vazio da armadura } \\ x_{a M} & \text { Reatância associada a } L_{a} M \\ x_{s l} & \text { Reatância de dispersão da armadura } \\ x_{d} & \text { Reatância própria de armadura de eixo } d \\ x_{q} & \text { Reatância própria de armadura de eixo } q \\ r_{s} & \text { Resistência de armadura } \\ \delta & \text { Ângulo de carga } \\ t_{e m} & \text { Torque eletromagnético } \\ T_{P} & \text { Torque máximo na região em que } \omega_{r}<\omega_{P} \\ i_{s d} & \text { Corrente de armadura de eixo } d \\ i_{s q} & \text { Corrente de armadura de eixo } q\end{array}$

\section{INTRODUÇÃO}

$\mathrm{O}$ uso de motores síncronos de ímãs permanentes em aplicações industriais vem crescendo expressivamente nos últimos anos. Esta nova tendência se deve principalmente à alta eficiência destes motores, que chega a ser de $2 \%$ a $3 \%$ maior que a dos motores de indução. A redução do peso e do volume também são fatores preponderantes para escolha destes motores (P\&D, 2007).

O uso de ímãs permanentes elimina as perdas que ocorrem no enrolamento de rotor das máquinas síncronas convencionais, possibilitando assim, o aumento da eficiência e do tempo de vida do motor que passa a funcionar com menores níveis de temperatura. Como o rotor é formado por ímãs de alta densidade de fluxo, consegue-se construir motores mais compactos e, consequentemente, com um menor sistema de ventilação.

Tradicionalmente, os motores síncronos de ímãs permanentes (MSIP) eram construídos com ímãs de ferrite ou samário-cobalto. Recentemente, materiais com alta densidade de fluxo (maior que 1,0 T) e elevada coercividade (maior que $7000 \mathrm{~A} / \mathrm{cm}$ ), como o Nedímio-ferroboro, possibilitaram a produção de motores para aplica- ção industrial. O uso destes ímãs permite a construção de motores com menor risco de desmagnetização e mais compactos, podendo ter uma redução de até $47 \%$ em seu volume e $36 \%$ em seu peso em relação a um motor de indução com a mesma potência (P\&D, 2007).

Os MSIP possuem um enrolamento de estator trifásico similar ao motor síncrono convencional e ímãs permanentes no lugar do enrolamento de campo. As características desses motores dependem basicamente do tipo dos ímãs e de como estes são montados no rotor. O motor de ímãs superficiais (MSIPS) tem os ímãs fixados na superfície do rotor através de um adesivo epóxi e o motor de ímãs interiores (MSIPI) possui os ímãs inseridos no rotor.

As saliências presentes no entreferro do MSIPI, devido à disposição dos ímãs no interior do rotor, fazem com que este motor possua uma elevada razão $x_{q} / x_{d}$ e, consequentemente, desenvolva o torque de relutância. Aproveitando esta parcela de torque através da adequada estratégia de controle aumenta-se a relação torque/corrente do motor, possibilitando o uso de um inversor de frequência de menor potência. Também é possível aproveitar o alto valor da razão $x_{q} / x_{d}$ para acionar o motor na região de redução de campo.

Fixar a componente de corrente $i_{s d}$ em zero para que somente a corrente $i_{s q}$ seja proporcional ao torque é a maneira mais simples de implementar um controle linear de torque do MSIPI. Entretanto, isto faz com que a parcela de torque de relutância seja desprezada e o sistema de acionamento apresente baixa eficiência. Por outro lado, para que o torque de relutância seja aproveitado pode-se empregar alguma estratégia de controle não linear como, por exemplo, os controladores adaptativos propostos por (Sozer and Torrey, 1998),(Bodson et al., 1993), (Caravani and Gennaro, 1998), (Marino et al., 1995), (Marques et al., 2006), (S. Laghrouche F. et al., 2003) e (Dias et al., 2008). Contudo, o projeto dos reguladores envolvido nestas estratégias é muito complexo e o modelo empregado depende de vários parâmetros do MSIPI.

Com o objetivo de tornar o sistema de acionamento menos sensível aos parâmetros do motor é implementado na região de torque constante um controle linear de torque baseado na curva de máximo torque por Ampère $(M T P A)$ que determina as referências de corrente $i_{s d}^{*} \mathrm{e}$ $i_{s q}^{*}$ em função da referência de torque $t_{e m}^{*}$ gerada pelo regulador de velocidade. Esta estratégia visa acionar o MSIPI com a máxima resposta dinâmica e a menor corrente de estator necessária para atender o torque 
requerido (Krishnan, 1993), (Vas, 1998), (Kim, 2004), (Øvrebø, 2004) e (Pan and Sue, 2005).

Na região de potência constante, a maneira mais simples de implementar um controle de redução de campo é aquela feita através da saturação dos reguladores de corrente, que ocorre em altas velocidades quando a tensão interna do MSIPI ultrapassa o valor de base (Vas, 1998). Contudo, esta estratégia possui baixa resposta dinâmica devido à limitação da ação dos reguladores de corrente. Para diminuir o tempo de resposta e, consequentemente, diminuir os picos de corrente durante os transitórios, utiliza-se um controle efetivo de corrente baseado na expressão da tensão terminal de armadura para acionar o MSIPI na região de potência constante (Krishnan, 1993), sendo que os resultados apresentados pelo autor são apenas de simulação. Com o objetivo de aumentar ainda mais a resposta, (Pan and Sue, 2005) definem uma faixa de operação faixa de operação intermediária entre as regiões de torque constante e potência constante, denominada região de redução de campo parcial, na qual utiliza-se o controle pela curva $M T P A$. A implementação de um acionamento para o controle de um motor síncrono de ímãs permanentes sem a utilização de um sensor de posição é reportado em (Imbuzeiro, 2008).

Neste artigo são apresentados e analisados os resultados de simulação e experimentais do controle linear de máximo torque de um MSIPI nas duas regiões do controle de velocidade: a de torque constante e a de potência constante (redução parcial e plena de campo). A contribuição está centrada na implementação em laboratório, com sucesso, da estratégia de controle simulada digitalmente por (Krishnan, 1993), e na proposta de (Pan and Sue, 2005), comentada anteriormente.

Na seção 2 é apresentado o modelo matemático do MSIPI; nas seções 3 e 4 são abordados o controle da velocidade com as estratégias de controle de máximo torque por Ampère e de redução de campo, sendo que nesta a operação se dá na região de potência constante e naquela, na de torque constante. Os resultados de simulação e experimentais são apresentados na seção 5 e as conclusões são colocadas na seção 6 .

\section{MODELO MATEMÁTICO DO MSIPI}

O modelo matemático do MSIPI é similar ao modelo do motor síncrono convencional com pólos salientes. O enrolamento de campo é substituído por ímãs permanentes, cujo eixo magnético coincide com o eixo direto do referencial síncrono fixado no rotor, isto é, o fluxo devido aos ímãs está inteiramente localizado no eixo di- reto, como ilustrado na Figura 1. A excitação estabelecida pelo ímã é modelada por uma fonte de corrente contínua de valor $i_{M}$ e o correspondente fluxo que concatena os enrolamentos da armadura é designado por $\psi_{r d}=\psi_{a M}=L_{a M} i_{M}$, sendo $L_{a M}$ a indutância mútua entre o enrolamento de estator e o fictício de rotor (ímã).

Se o modelo matemático do MSIPI estiver descrito em p.u., todas as indutâncias são numericamente iguais as suas correspondentes reatâncias e, portanto, tem-se $x_{a M}=L_{a M}$ p.u. Consequentemente, o fluxo do ímã que concatena os enrolamentos de armadura é dado por

$$
\psi_{a M}=x_{a M} i_{M}
$$

Portanto, o vetor espacial deste fluxo descrito no sistema de coordenadas estacionário é

$$
\bar{\psi}_{a M}=x_{a M} i_{M} e^{j \theta_{r}} \quad \text { p.u. }
$$

cujo o ângulo elétrico do rotor $\left(\theta_{r}\right)$ é definido por

$$
\theta_{r}=\omega_{r} t+\theta_{r 0}
$$

e $\omega_{r}$ é a velocidade síncrona do rotor descrita em radianos elétricos.

O vetor espacial da tensão terminal de armadura no referencial estacionário é

$$
\bar{V}_{s, \alpha \beta}=r_{a} \bar{I}_{s, \alpha \beta}+\frac{d \bar{\psi}_{s, \alpha \beta}}{d t}
$$

Após multiplicar a Equação 4 pelo operador $e^{-j \theta_{r}}$ e fazer as devidas manipulações, obtém-se a expressão do vetor espacial da tensão de armadura no referencial síncrono,

$$
\bar{V}_{s, d q}=r_{s} \bar{I}_{s, d q}+\frac{d \bar{\psi}_{s, d q}}{d t}+j \omega_{r} \bar{\psi}_{s, d q}
$$

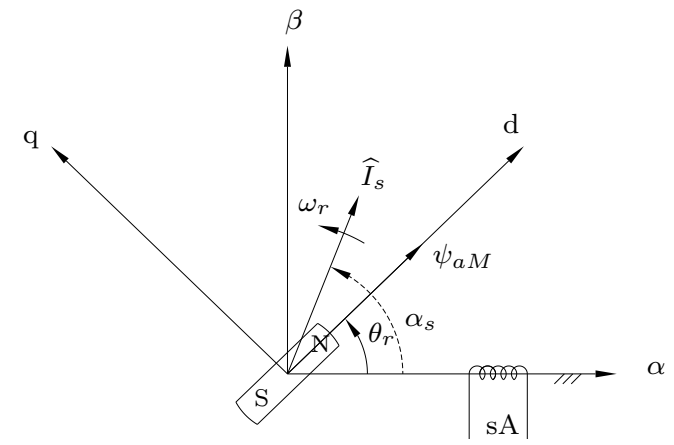

Figura 1: Vetores espaciais da corrente de armadura e fluxo concatenado produzido pelos ímãs. 
que decomposta nos eixos direito e quadratura resulta em

$$
\begin{aligned}
& v_{s d}=r_{s} i_{s d}+\frac{d \psi_{s d}}{d t}-\omega_{r} \psi_{s q} \\
& v_{s q}=r_{s} i_{s q}+\frac{d \psi_{s q}}{d t}+\omega_{r} \psi_{s d}
\end{aligned}
$$

onde as componentes do vetor $\bar{\psi}_{s d q}$ são

$$
\begin{gathered}
\psi_{s d}=x_{d} i_{s d}+\psi_{a M} \\
\psi_{s q}=x_{q} i_{s q}
\end{gathered}
$$

com $x_{d}$ e $x_{q}$ sendo as reatâncias de eixo direto e em quadratura, respectivamente. Ao substituir, convenientemente, estas expressões das componentes de fluxo no conjunto de equações das tensões, e considerando o regime permanente, obtêm-se as seguintes equações de tensão:

$$
\begin{gathered}
v_{s d}=r_{s} i_{s d}-x_{q} i_{s q} \\
v_{s q}=r_{s} i_{s q}+x_{d} i_{s d}+E_{a M}
\end{gathered}
$$

Ao substituir as componentes do fluxo de armadura equações 8 e 9-, na expressão do conjugado

$$
t_{e m}=\psi_{s d} i_{s q}-\psi_{q} i_{s d}
$$

obtém-se a seguinte expressão do torque eletromagnético em função das correntes $i_{s d}$ e $i_{s q}$ e do fluxo dos ímãs $\psi_{a M}$ :

$$
t_{e m}=\psi_{a M} i_{s q}+\left(x_{d}-x_{q}\right) i_{s d} i_{s q}
$$

\section{CONTROLE DE MÁXIMO TORQUE POR AMPÈRE - REGIÃO DE TORQUE CONSTANTE}

Mediante a orientação do fluxo de rotor é possível controlar o torque desenvolvido pelo MSIPI por meio das correntes $i_{s d}$ e $i_{s q}$. Para que o torque de relutância seja adicionado ao torque devido aos ímãs é necessário se ter uma corrente de armadura que produza um fluxo no entreferro em contraposição ao fluxo dos ímãs. Isto é feito através do avanço do fasor da corrente de armadura a partir do eixo em quadratura, como pode ser observado no diagrama fasorial da Figura 2. O avanço do ângulo de carga $\delta$ produz uma componente da reação de armadura de eixo em quadratura maior do que aquela de eixo direto, ou seja: $x_{q} I_{s q}>x_{d} I_{s d}$; assim, o fasor $\hat{V}_{s}$ mantém-se adiantado em relação ao fasor $\hat{I}_{s}$. Além do fator de potência indutivo, outra característica da região de torque constante é o fato do MSIPI operar com subexcitação, pois como observado no diagrama fasorial, tem-se $E_{a M}<v_{s q}$.

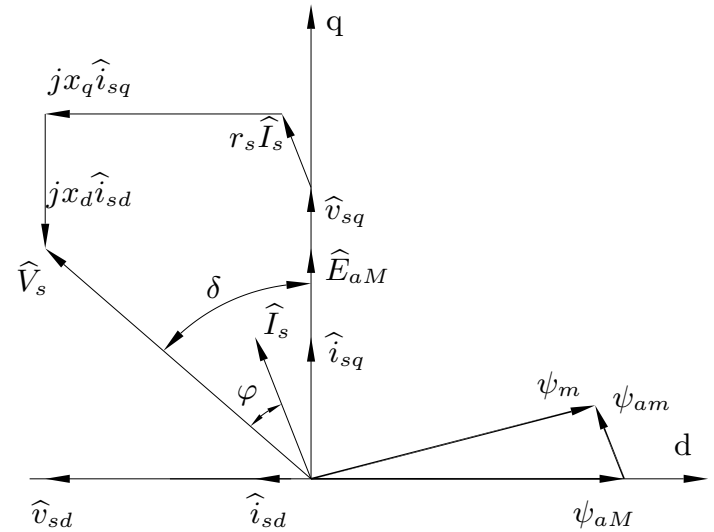

Figura 2: Diagrama fasorial na região de torque constante: $E_{a M}<v_{s q}$.

Com base na curva de máximo torque por Ampère, desenvolve-se uma estratégia de controle linear de torque para acionar o MSIPI na região de torque constante. Esta estratégia consiste em obter as funções que determinam as referências de corrente $i_{s d}^{*}=f\left(t_{e m}^{*}\right)$ e $i_{s q}^{*}=f\left(t_{e m}^{*}\right)$, a partir do sinal $t_{e m}^{*}$ gerado pelo regulador de velocidade. Estas funções são implementadas nos blocos de função $F_{1}$ e $F_{2}$, como pode ser observado na Figura 3.

A partir da Equação 13 são obtidas as hiperbóles de torque representadas na Figura 4. A curva MTPA é formada pelos pontos mais próximos da origem que correspondem à mínima corrente $I_{s}$ necessária para atender o torque requerido.

Ao substituir a Equação 13 na expressão do módulo da corrente $I_{s}$, dada por

$$
I_{s}=\sqrt{i_{s d}^{2}+i_{s q}^{2}}
$$

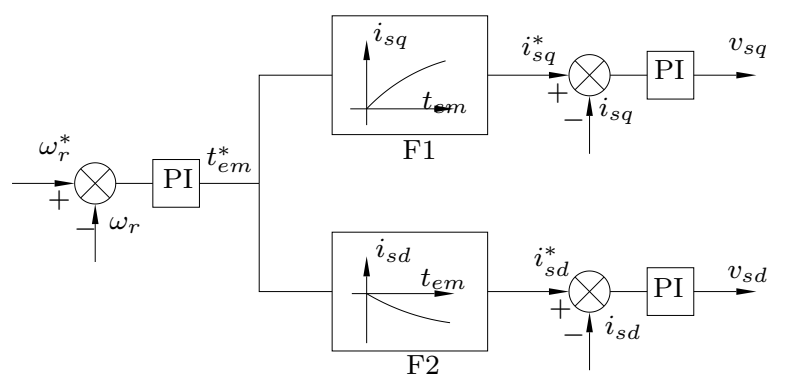

Figura 3: Diagrama de blocos do controle na região de torque constante. 


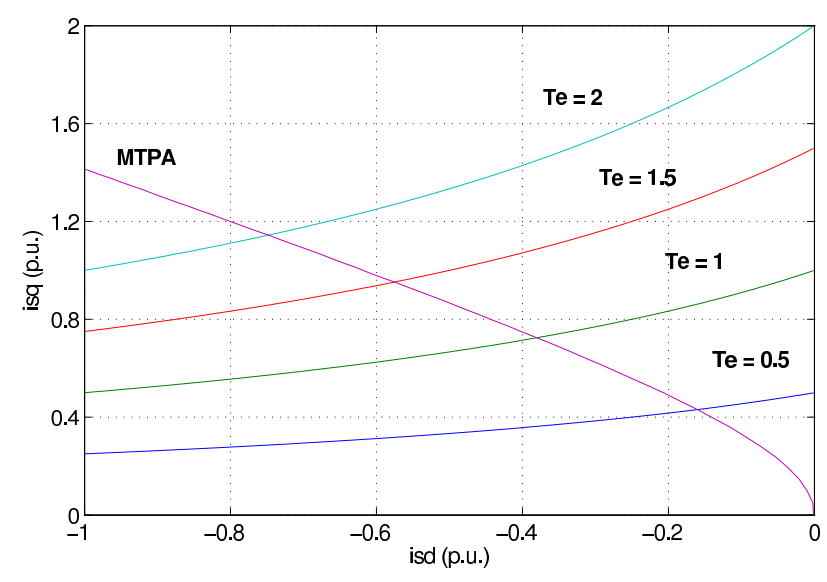

Figura 4: Hipérboles de torque e curva MTPA.

obtém-se,

$$
I_{s}=\sqrt{i_{s d}^{2}+\left[\frac{t_{e m}}{\psi_{a M}+\left(x_{d}-x_{q}\right) i_{s d}}\right]^{2}}
$$

O mínimo valor de $I_{s}$ necessário para atender o torque requerido é obtido através da equação diferencial

$$
\frac{d I_{s}}{d i_{s d}}=0
$$

A solução da Equação 16 resulta na seguinte função polinomial, que é implementada no bloco $F_{1}$, mostrado na Figura 3:

$$
\begin{aligned}
& 2 X_{d q}^{3} i_{s d}^{*}+6 \psi_{a M} X_{d q}^{2} i_{s d}^{*}{ }^{3}+ \\
& +6 \psi_{a M}^{2} X_{d q} i_{s d}^{*}{ }^{2}+2 \psi_{a M}^{3} i_{s d}^{*}-2 t_{e m}^{*}{ }^{2} X_{d q}=0
\end{aligned}
$$

na qual $X_{d q}=x_{d}-x_{q}$. Ao se explicitar a corrente $i_{s d}^{*}$ na Equação 13 e substituindo-a na Equação 17, obtémse a função implementada no bloco $F_{2}$, mostrado na Figura 3:

$$
2 X_{d q}^{4} i_{s q}^{*}{ }^{5}+2 \psi_{a M} t_{e m}^{*} X_{d q}^{2} i_{s q}^{* 2}-2 t_{e m}^{*}{ }^{2} X_{d q}^{2} i_{s q}^{*}=0
$$

\section{CONTROLE DE REDUÇÃO DE CAMPO - REGIÃO DE POTÊNCIA CONSTANTE}

A rápida resposta dinâmica obtida com o controle $M T P A$ não se repete para o controle de redução de campo, por que, acima de 1 p.u. de velocidade, a ação dos reguladores de corrente é limitada para que a tensão terminal de estator se mantenha constante. Para que não ocorra a saturação dos reguladores é necessário reduzir o fluxo de magnetização através do controle da

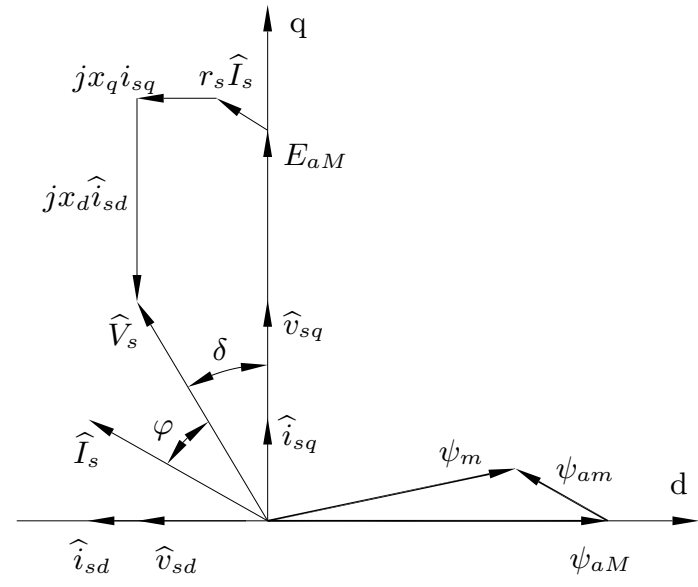

Figura 5: Diagrama fasorial na região de redução plena: $E_{a M}>$ $v_{s q}$.

reação de armadura. O avanço do ângulo $\delta$, obtido com o controle da reação de armadura, gera um fluxo que atua em contraposição ao fluxo concatenado devido aos ímãs, o que resulta em um menor fluxo de magnetização de entreferro $\psi_{m}$, como pode ser visto no diagrama fasorial da Figura 5. Também é possível notar, ao se analisar o diagrama, que, para aumentar a componente de corrente $i_{s d}$, é necessário limitar a corrente $i_{s q}$. Assim, à medida que o motor acelera, o ângulo $\delta$ aumenta para permitir que a operação se dê na região de redução de campo, mas o torque desenvolvido pelo MSIPI é reduzido para que o fasor $\hat{I}_{s}$ não ultrapasse o valor nominal do inversor de frequência. Com isso, a tensão terminal de estator é limitada em 1 p.u. e o MSIPI é acionado com potência constante. Nota-se também que o controle nesta região é caracterizado pelo fator de potência capacitivo e pela superexcitação do motor, pois $E_{a M}>v_{s q}$.

O diagrama fasorial representando na Figura 6 indica que o adequado controle da reação de armadura acima da velocidade de base ocorre quando o módulo do fasor reação de armadura $\left(x_{d} i_{s d}+x_{q} i_{s q}\right)$ é igual ao módulo do fasor tensão de armadura gerada pelos ímãs $\left(E_{a M}\right)$, mantendo a tensão terminal de estator $\left(V_{s}\right)$ no seu valor nominal. Quando $E_{a M}>\left(x_{d} i_{s d}+x_{q} i_{s q}\right)$, ter-se-ia $V_{s}>1$ p.u., mas o controle limita esta tensão em 1 p.u.; se $E_{a M}<\left(x_{d} i_{s d}+x_{q} i_{s q}\right)$, a tensão de terminal seria menor que 1.0 p.u., isto é, $V_{s}<1$ p.u., e, consequentemente, o motor ficaria com excesso de reativos, apresentando, então, baixa eficiência.

De acordo com a Figura 7, à medida que o MSIPI acelera na região de redução de campo, o valor da corrente $I_{s}$ é limitada pelo aumento da velocidade e seu valor é 
dado por curvas na forma de elipses. Para uma dada tensão de magnitude $V_{s}$, estas elipses são determinadas pela seguinte expressão:

$$
i_{s q}^{2}=\frac{V_{s}^{2}-\omega_{r}^{2}\left(x_{d} i_{s d}+\psi_{a M}\right)^{2}}{\omega_{r}^{2} x_{q}^{2}}
$$

Observa-se na Figura 7 que o máximo torque $\left(T_{P}\right)$ obtido pelo controle $M T P A$ não pode ser mantido acima da velocidade $\omega_{P}$. Acima desta velocidade a trajetória da corrente é transferida da curva $M T P A$ para a circunferência que limita a corrente de armadura em 1 p.u.

A elipse que representa a velocidade de base é aquela que intercepta a origem dos eixos $d$ e $q$. Portanto, ao substituir $i_{s d}=i_{s q}=0$ na Equação 19 tem-se a velocidade de base do motor

$$
\omega_{b}=\frac{V_{s}}{\psi_{a M}}
$$

O MSIPI atinge a máxima velocidade quando está operando com torque igual a zero $\left(i_{s q}=0\right)$ e potência nominal com $i_{s d}=-1,0$ p.u. e $V_{s}=1,0$ p.u. Reescrevendo a Equação 19 para estas condições tem-se,

$$
\omega_{\operatorname{máx}}=\frac{1}{\psi_{a M}-x_{d}}
$$

Apesar de o MSIPI operar na região de redução de campo na faixa de velocidade $\omega_{P}<\omega_{r}<\omega_{b}$, se $V_{s}<1,0$ p.u., o motor ainda é acionado com base na conhecida curva $M T P A$. Ao estender o acionamento pela curva $M T P A$, diminui-se o tempo de resposta, bem como os picos de corrente durante os transitórios, devido ao fato de os reguladores de corrente terem maior margem de ação. Esta faixa de velocidade é denominada região de redução parcial e a faixa $\omega_{b}<\omega_{r}<\omega_{\text {máx }}$ é a região de redução pleno. Nota-se no diagrama fasorial da Figura 8 que, embora o fator de potência seja indutivo, o MSIPI opera com superexcitação na região de redução parcial, pois $E_{a M}>v_{s q}$. O máximo valor de $V_{s}$, determinado pela tensão do barramento de corrente contínua

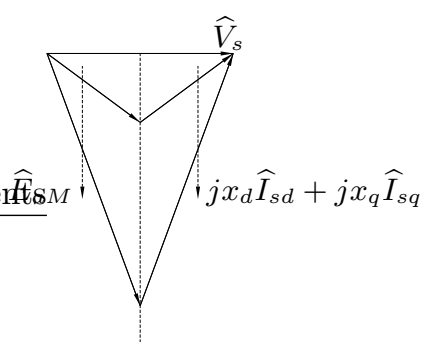

Figura 6: Controle ótimo da reação de armadura.

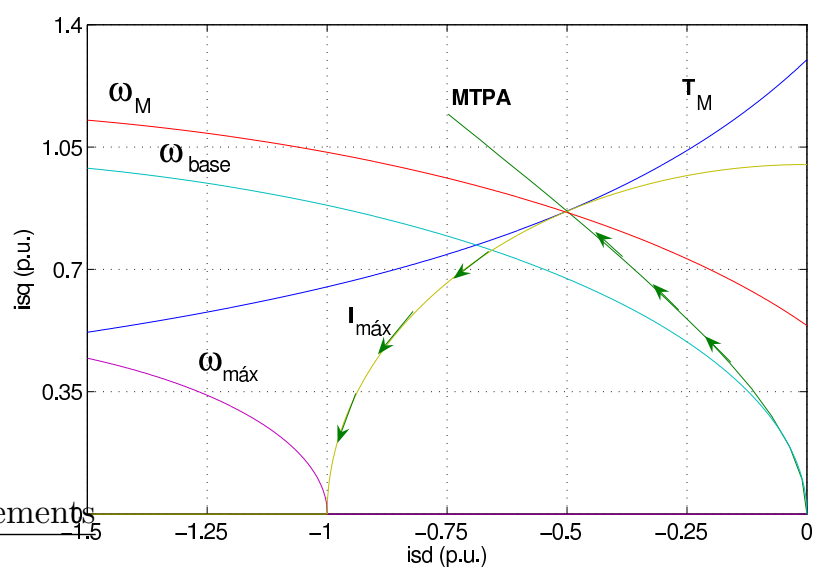

Figura 7: Curva $M T P A$, hipérboles de torque, elipses de tensão e circunferência de máxima corrente.

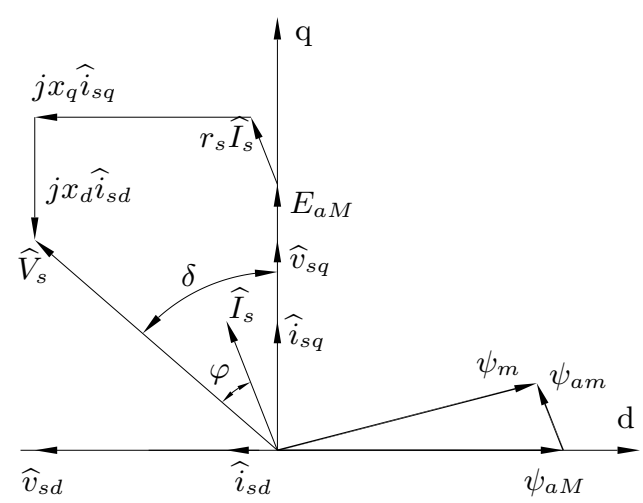

Figura 8: Diagrama fasorial na região de redução parcial: $E_{a M}>v_{s q}$.

do inversor de frequência, é dado por

$$
V_{s}=\sqrt{v_{s d}^{2}+v_{s q}^{2}}
$$

Substituindo as Equações 6 e 7, particularizadas para o regime permanente e desconsiderando $r_{s}$, a partir da Equação 22 obtém-se,

$$
V_{s}^{2}=\omega_{r}^{2}\left[\left(x_{d} i_{s d}+\psi_{a M}\right)^{2}+\left(x_{q} i_{s q}\right)^{2}\right]
$$

Ao se combinar a Equação 23 à expressão da corrente $i_{s q}$, dada por

$$
i_{s q}=\sqrt{I_{s}^{2}-i_{s d}^{2}}
$$

obtém-se a expressão final da tensão de terminal $V_{s}$ em função das correntes $I_{s}$ e $i_{s d}$ e da velocidade $\omega_{r}$ :

$$
V_{s}^{2}=\omega_{r}^{2}\left[\left(x_{d} i_{s d}+\psi_{a M}\right)^{2}+x_{q}^{2}\left(I_{s}^{2}-i_{s d}^{2}\right)\right]
$$


Para que o MSIPI possa operar com potência nominal durante a redução de campo, o valor do fluxo $\psi_{a M}$, da tensão $V_{s}$ e da corrente $I_{s}$ são mantidos em 1 p.u.. Consequentemente, a Equação 25 torna-se função apenas das variáveis $\omega_{r}$ e $i_{s d}$

$$
a i_{s d}^{2}+b i_{s d}+c=\omega_{r}^{2}
$$

onde as constantes $a, b$ e $c$ são

$$
\begin{aligned}
& a=x_{d}^{2}-x_{q}^{2} \\
& b=2 x_{d} \\
& c=1+x_{q}^{2}
\end{aligned}
$$

Isolando $i_{s d}$ na Equação 26 obtém-se a componente de corrente $i_{s d}^{*}$ em função da velocidade do rotor

$$
i_{s d}=\frac{\left(-b+\sqrt{b^{2}-4 a\left(c-\frac{1}{\left(\omega_{r}\right)^{2}}\right)}\right)}{2 a}
$$

que é utilizada para aplicar uma corrente $i_{s d}$ negativa no MSIPI de maneira a enfraquecer o fluxo de magnetização e permitir que ele seja acionado com tensão constante na região de redução de campo.

Observa-se no diagrama de blocos da Figura 9 que a referência $i_{s d}^{*}$, gerada pela função $i_{s d}^{*}=f\left(\omega_{r}\right)$, determina a máxima corrente $i_{\text {sqm }}$ com a qual o MSIPI pode operar em velocidades acima da velocidade de base $\omega_{b}$ e, consequentemente, o máximo torque eletromagnético $t_{\max }$ que o MSIPI pode desenvolver nessas condições. Portanto, a referência de torque $t_{e m}^{*}$ gerada pelo regulador de velocidade é comparada com $t_{\max }$ a partir da seguinte operação lógica:

$$
\begin{array}{ll}
\text { se } t_{e m}^{*}>t_{\max }, & \text { então } t_{\text {max }}^{*}=t_{\max } \\
\text { se } t_{\text {em }}^{*}<t_{\max }, & \text { então } t_{\max }^{*}=t_{\text {em }}^{*}
\end{array}
$$

para que seja determinado o máximo torque que o MSIPI pode desenvolver. Finalmente, ao substituir $t_{e m}^{*}$ e $i_{s d}^{*}$ na Equação 13, obtém-se o valor da referência $i_{s q}^{*}$.

\section{RESULTADOS}

O modelo matemático do sistema de controle proposto é mostrado no diagrama de blocos da Figura 10. Os resultados de simulação obtidos não consideram a modulação PWM. Esta simplificação não invalida a comparação com os resultados experimentais, pois, segundo (Pillay and Krishnan, 1989), se o atraso da modulação, que é aproximadamente um período da frequência de chaveamento, for menor que um décimo da constante de tempo da armadura, a resposta dinâmica do sistema de controle não será afetada. Como a constante de tempo do MSIPI utilizado neste trabalho, cujos dados estão descritos na Tabela 1, está em torno de 30 ms e o período da frequência de chaveamento utilizada para obter os resultados experimentais é $0,2 \mathrm{~ms}$, os efeitos da modulação são desprezíveis. A montagem experimental utiliza um microprocessador SH7085-RENESAS, acoplado a placa de condicionamento de sinais que comanda o driver dos IGBTs de um inversor de frequência, modelo CFW11WEG. A leitura das variáveis de controle é feita através

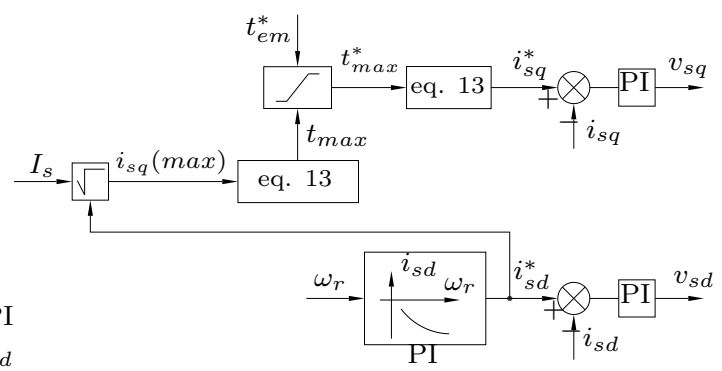

Figura 9: Diagrama de blocos do controle na região de redução de campo.

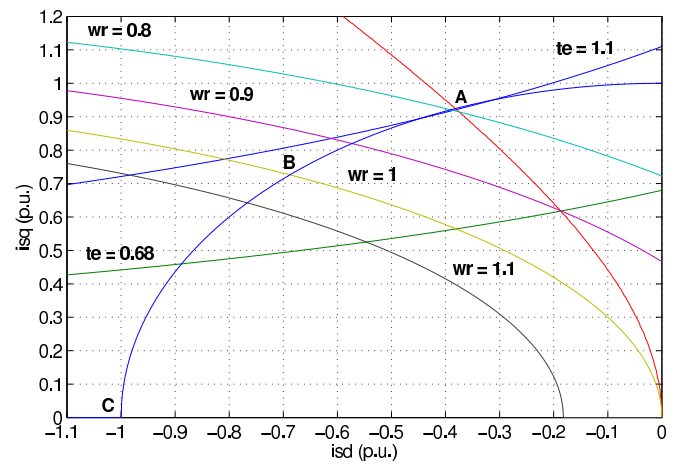

(a) Diagrama circular

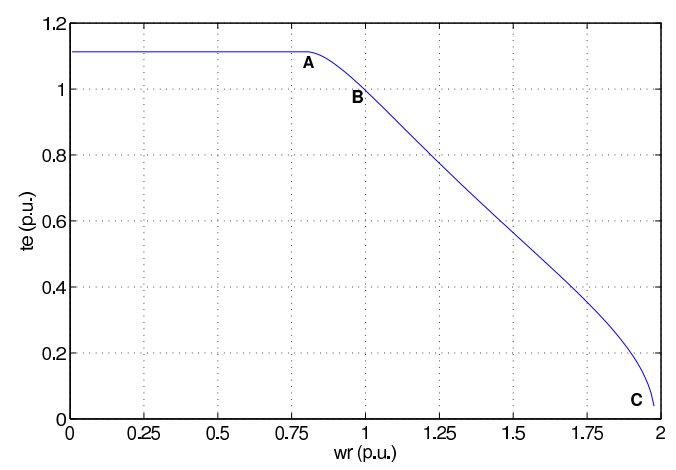

(b) Característica de torque

Figura 11: Diagrama e característica de torque do MSIPI. 


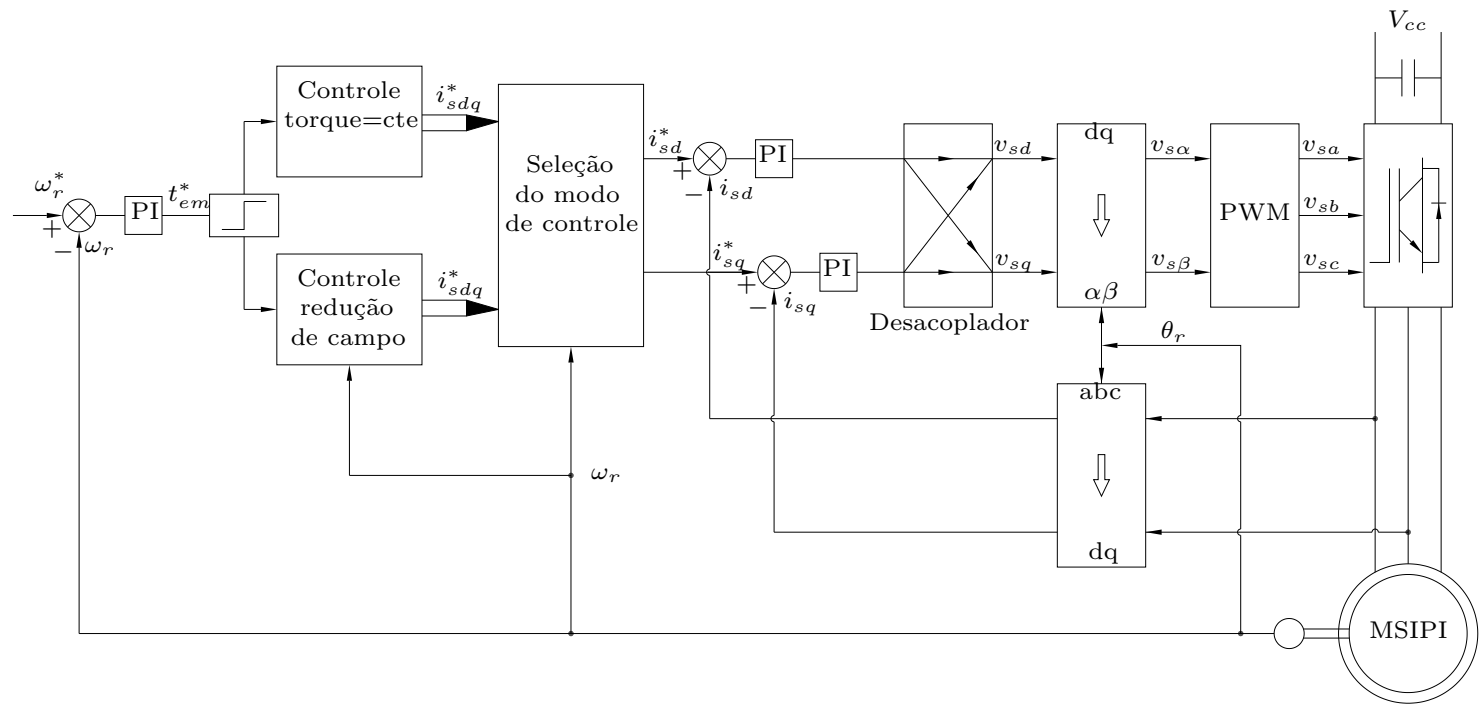

Figura 10: Diagrama de blocos do controle do MSIPI.

de um conversor A/D com resolução de 12 bits acoplado à placa de condicionamento. $\mathrm{O}$ inversor de frequência aciona um MSIPI-WEG acoplado a um dinamômetro ALPHA 240/AVL. A realimentação de velocidade é feita através de um encoder incremental de 1.024 ppr.

A curva-MTPA do MSIPI utilizado nos testes, apresentada em traço vermelho na Figura 11(a), determina que o motor pode ser acionado com uma relação torque/corrente de 1,1 N.m/A com velocidades angulares de até 0,8 p.u., como indica o ponto $A$ mostrado nas Figuras 11(a) e 11(b). Se o MSIPI estiver sendo acionado com uma tensão terminal de armadura menor que o valor de base na faixa de velocidade limitada pelos pontos $A$ e $B$, estende-se o controle pela curva- $M T P A$. $\mathrm{O}$ valor de base utilizado para normalizar a velocidade é aquele obtido com a tensão interna $E_{a M}=1,0$ p.u. A partir desta velocidade, que é representada pelo ponto $B$ nas Figuras 11(a) e 11(b), o MSIPI é acionado apenas mediante o controle de redução de campo. O ponto $C$ representa a máxima velocidade de operação.

No primeiro teste, o MSIPI é acelerado de 0 a 0,9 p.u., segundo um perfil de velocidade em forma de rampa linear, com uma carga de torque igual a 0,68 p.u. $O$ tempo de aceleração é ajustado em $7,5 \mathrm{~s}$ para que tanto o regulador de velocidade quanto os reguladores de corrente não entrem em saturação, e a resposta dinâmica do sistema fique apenas condicionada ao método de controle. Apesar da velocidade utilizada neste teste estar na região de redução de campo, o MSIPI é acionado segundo a curva-MTPA com um valor de carga que não provoca a saturação dos reguladores de corrente. $\mathrm{O}$
MSIPI pode ser acionado de acordo com a curva- $M T P A$ com 0,9 p.u. de velocidade, desde que o torque não seja superior a 0,68 p.u, caso contrário, é feita a transição para o controle de redução de campo para que a tensão terminal de armadura seja limitada ao valor de base.

Nota-se, nos gráficos referentes às correntes $i_{s d}$ e $i_{s q}$ da Figura 12, que os seus valores em regime convergem para os valores determinados pelo diagrama circular da Figura 11(a), na qual este ponto de operação é a intersecção entre a curva- $M T P A$ e a elipse relativa à velocidade igual a 0,9 p.u.. O acionamento segundo a curva-MTPA garante ao sistema de controle uma relação torque/corrente de 1,1 N.m/A. A substituição dos valores de $i_{s d}$ e $i_{s q}$ na Equação 13 resulta nos valores de 0,6 p.u. e 0,08 p.u., para o torque devido aos ímãs e à variação da relutância, respectivamente. A soma destas componentes valida o valor de torque determinado segundo o diagrama da Figura 11(b).

De acordo com o gráfico de tensão da Figura 13, concluise que este é o máximo torque com o qual o MSIPI pode ser acionado com a velocidade de 0,9 p.u., pois nesta condição a tensão terminal de armadura é igual ao valor de base, e portanto, acima desta velocidade o MSIPI acelera com potência constante mediante o controle da reação de armadura.

A partir da resposta dinâmica das correntes e do torque, mostradas na Figura 12(a) e 12(b), constata-se que as características dos resultados de simulação se repetem, quase integralmente, nos resultados experimentais, com exceção do início da aceleração. Esta divergência 
explica-se, essencialmente, pela limitação de torque imposta pela curva de carga do dinamômetro para velocidades abaixo de $200 \mathrm{rpm}$.

No segundo teste, o MSIPI é controlado segundo a técnica de redução parcial de campo, pois o torque exigido nesta condição é maior do que aquele dado pel Figura 11(b). Portanto, o MSIPI acelera de acordo com curva- $M T P A$, até a velocidade igual a 0,8 p.u., com aproximadamente 1,3 p.u. de torque (carga + inércia), valor para o qual atinge-se 1 p.u. de tensão. A partir deste ponto de operação, o MSIPI continua a acelerar, segundo a estratégia de redução de campo, com tensão de armadura constante. Observase no gráfico de velocidade da Figura 14(a) que, nestas condições, é necessário ajustar a rampa de aceleração com um tempo duas vezes maior do que a do primeiro teste, para que os reguladores de corrente não saturem.
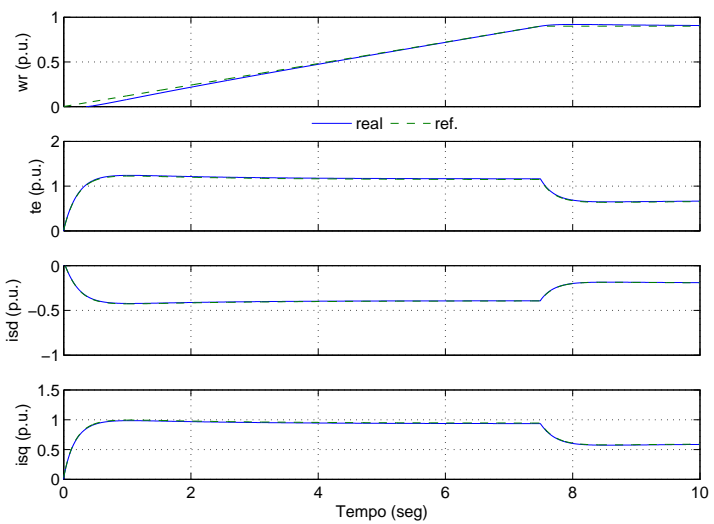

(a) Simulação
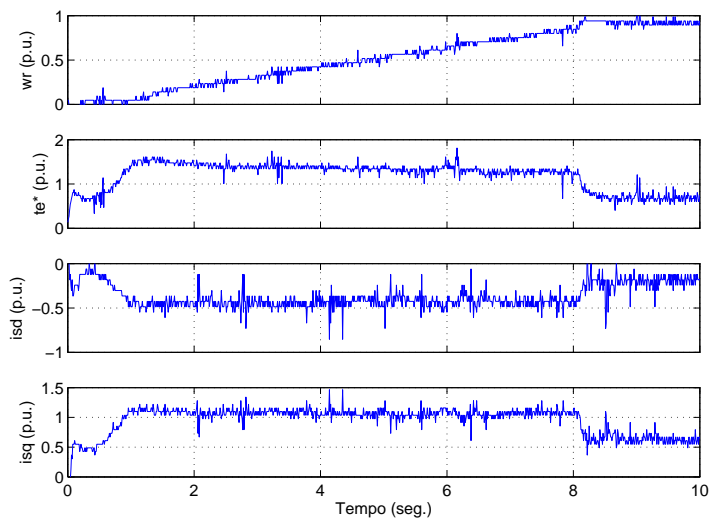

(b) Experimental

Figura 12: Velocidade, torque e correntes $i_{s d}$ e $i_{s q}$ para 0,9 p.u. de velocidade e 0,68 p.u. de torque.
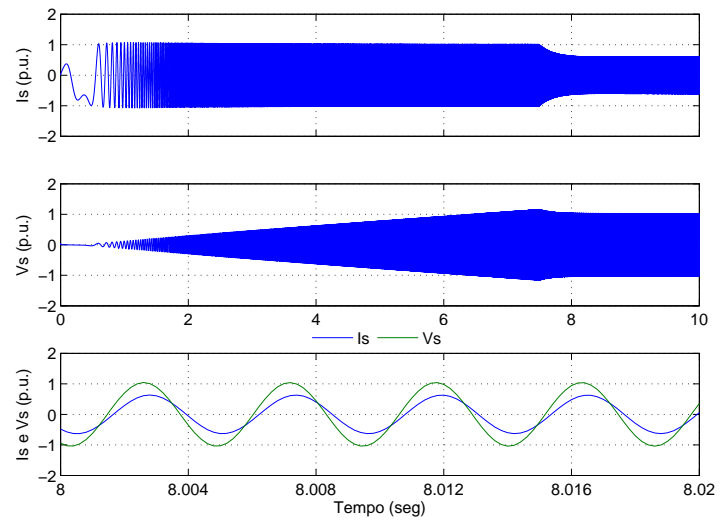

(a) Simulação
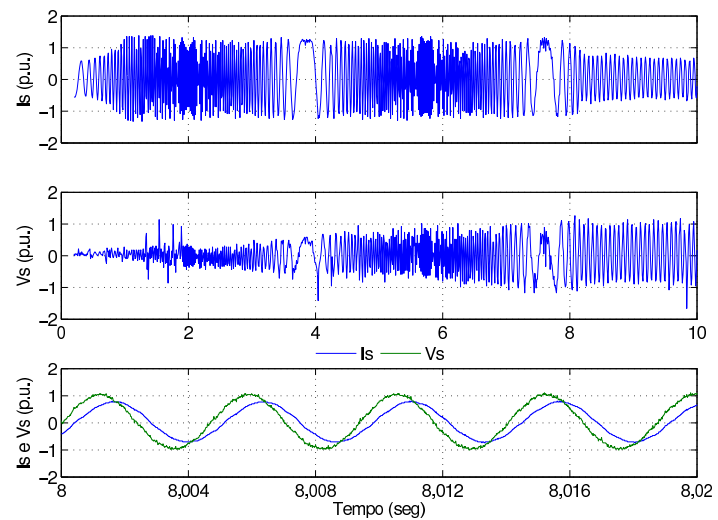

(b) Experimental

Figura 13: Corrente e tensão de fase para 0,9 p.u. de velocidade e 0,68 p.u. de torque.

A operação com redução de campo provoca uma redução de $3 \%$ na relação torque/corrente em comparação ao teste anterior. Esta relação é obtida com 0,84 p.u. de torque devido aos ímãs e 0,23 p.u. de torque de relutância. A soma destas componentes converge para o valor de 1,07 p.u., determinado pela característica de torque apresentada na Figura 11(b). A redução da relação torque/corrente é causada pelo avanço da corrente de armadura em relação ao eixo de quadratura.

Os resultados da Figura 15(a), mostram que é possível gerar um torque de até 1,07 p.u., mediante o enfraquecimento de campo, sem que a tensão de armadura ultrapasse o valor de base. O aumento do fator de potência, observado com a diminuição do ângulo de potência, é consequência do aumento em módulo da componente de corrente $i_{s d}$.

Para verificar o controle na região de redução plena, a velocidade e torque são tomados iguais a 1,1 e 0,9 p.u., respectivamente. Nesta condição de operação utiliza- 

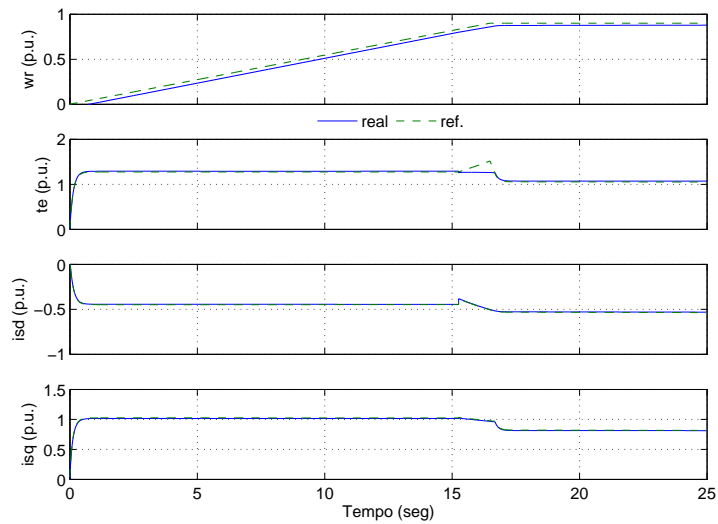

(a) Simulação
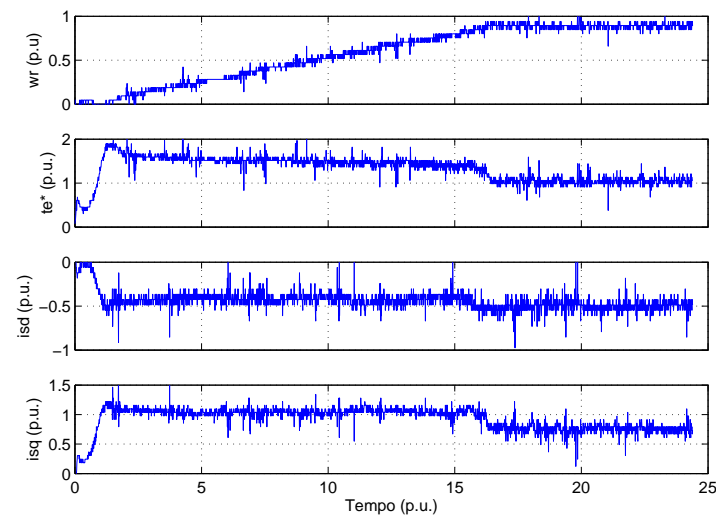

(b) Experimental

Figura 14: Velocidade, torque e correntes $i_{s d}$ e $i_{s q}$ para 0,9 p.u. de velocidade e 1,07 p.u. de torque.

se impreterivelmente o controle de redução de campo, pois $E_{a M}>1$ p.u.. Verifica-se também no diagrama da Figura 11(a) que não é possível utilizar o controle $M T P A$, pois a elipse obtida para velocidade igual a 1,1 p.u. não intersepta a curva $M T P A$. Portanto, como observado no teste anterior, o MSIPI também acelera até 0.8 p.u. de velocidade com aproximadamente 1.3 p.u. de torque $($ carga + inércia) através da curva MTPA, quando atinge-se 1 p.u. de tensão. Neste ponto ocorre a transição entre os métodos de controle, e o MSIPI é acionado através do controle de redução de campo, com tensão de armadura constante.

A partir do gráfico de torque da Figura 16 fica evidente que a transição dos métodos de controle é feita no momento em que ocorre a limitação de torque. A partir deste ponto a corrente $i_{s d}$ é determinada pela função $i_{s d}^{*}=f\left(\omega_{r}\right)$ a fim de manter a tensão terminal de armadura constante.
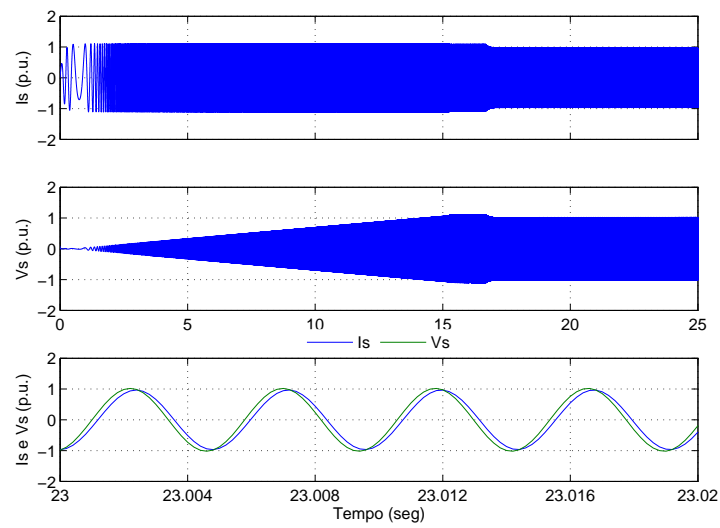

(a) Simulação
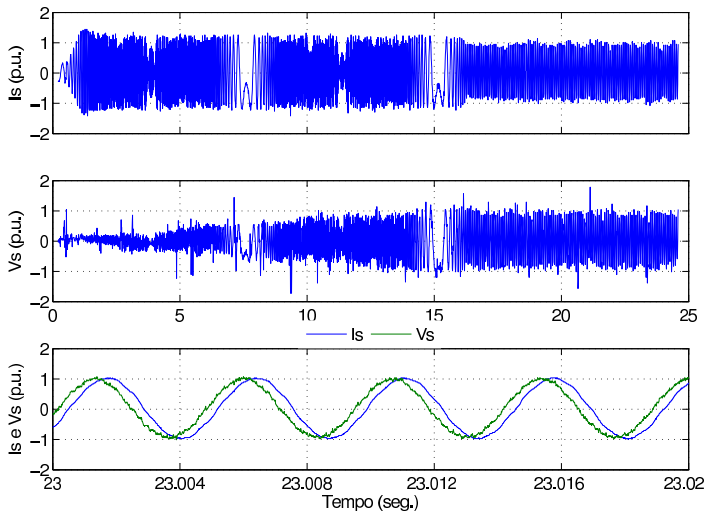

(b) Experimental

Figura 15: Corrente e tensão de fase para 0,9 p.u. de velocidade e 1,07 p.u. de torque.

A relação torque/corrente, obtida com este nível de redução de campo, é $20 \%$ menor do que a do teste feito com a curva-MTPA, e o avanço de corrente necessário para manter a potência constante implica em uma redução de $62 \%$ na resposta dinâmica em relação ao primeiro teste, ou, de forma alternativa, no aumento de aproximadamente $160 \%$ no tempo de resposta. .

Os valores de $i_{s d}$ e $i_{s q}$ obtidos nos gráficos de corrente da Figura 16 condizem com a intersecção entre a elipse obtida para 1,1 p.u. de velocidade e a circunferência de máxima corrente do diagrama circular.

\section{CONCLUSÃO}

Neste artigo é proposto o controle linear de torque de um MSIPI que utiliza a curva- $M T P A$, com o objetivo de aproveitar o torque de relutância e operar o motor com a máxima relação torque/corrente na região de torque constante. Isto possibilita o uso de um inversor de 

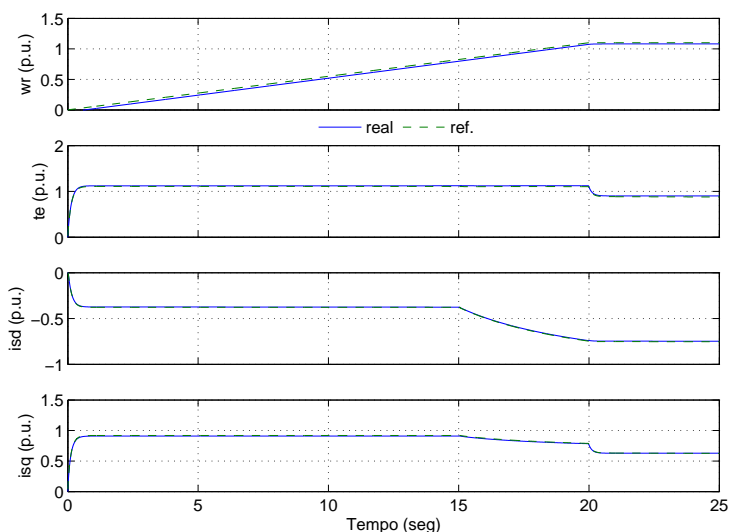

(a) Simulação
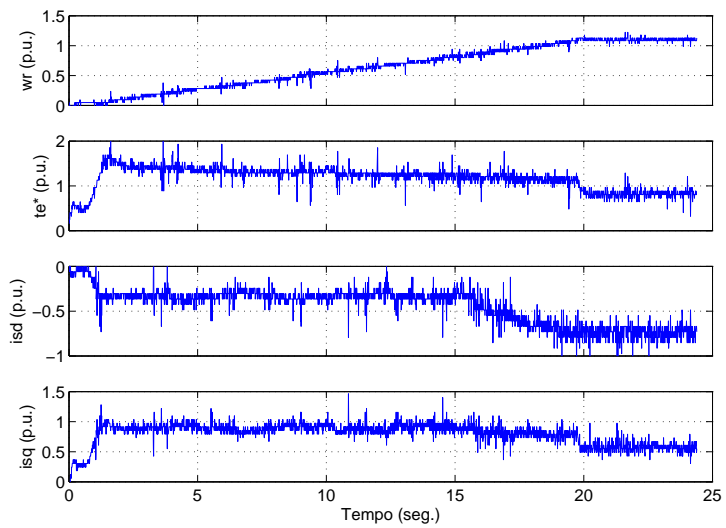

(b) Experimental

Figura 16: Velocidade, torque e correntes $i_{s d}$ e $i_{s q}$ para 1,1 p.u. de velocidade e 0,9 p.u. de torque.

frequência de menor potência na montagem experimental. Verifica-se, a partir dos resultados experimentais, que a extensão do controle, segundo a curva-MTPA na região de redução parcial de campo, diminui o tempo de resposta e, consequentemente, os picos de corrente durante os transitórios. Os resultados também mostram que o controle da reação de armadura permite que o MSIPI opere com velocidades de até duas vezes do seu valor nominal, porém, com relação torque/corrente reduzida.

\section{REFERÊNCIAS}

Bodson, M., Nonotnak, R. and Rekowski, R. (1993). High performance nonlinear control of a permanent magnet stepper motor, IEEE Transactions Control Systems Technology.

Caravani, P. and Gennaro, S. D. (1998). Robust control of synchronous motors with non-linearities and
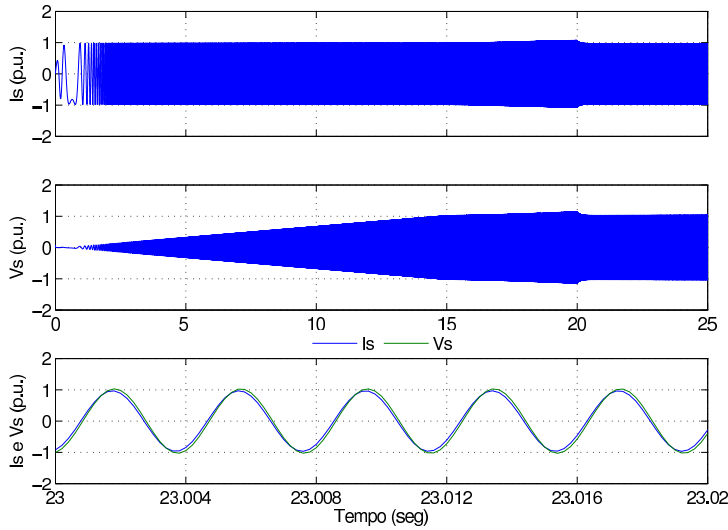

(a) Simulação
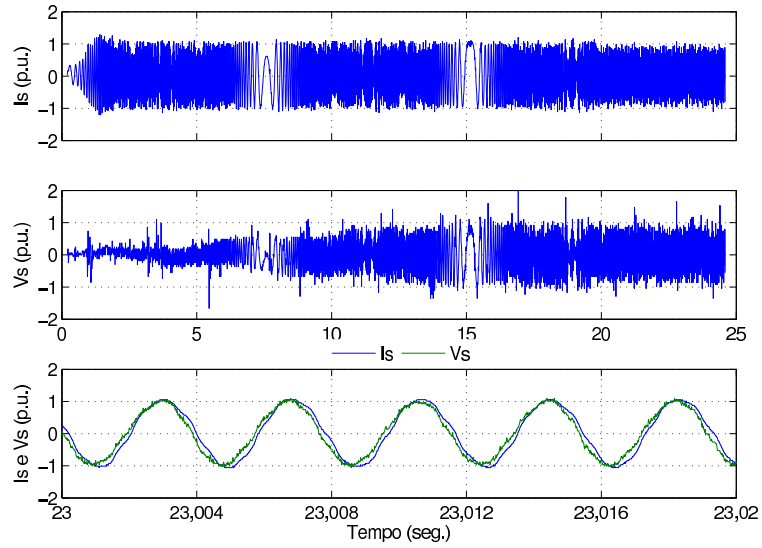

(b) Experimental

Figura 17: Corrente e tensão de fase para 1,1 p.u. de velocidade e 0,9 p.u. de torque.

parameters uncertainties, Automatica .

Dias, M., de S. Marques, L. C., Nied, A. and de Oliveira, J. (2008). Controle robusto do motor síncrono de ímãs permanentes baseado na abordagem dos modos deslizantes de segunda ordem, 8th INDUSCON

Imbuzeiro, R. S. S. (2008). Controle de um motor síncrono de Ímãs permanentes sem sensor de posição utilizando dsp, tese de mestrado, COPPE/UFRJ .

Kim, H. (2004). On-line parameter estimation, current regulation, and self-sensing for IPM synchronous machine drives, $\mathrm{PhD}$ thesis, University of Wisconsin-Madison.

Krishnan, R. (1993). Control and operation of pm synchronous motor drives in the field - weakening region, IEEE IECON93. 
Marino, R., Tomei, P. and Peresada, S. (1995). Nonlinear adaptive control of permanent magnet step motors, Automatica .

Marques, L. C. S., Fergütz, M. and Pieri, E. R. D. (2006). Controle não linear do servomotor ca de ímãs permanentes com robustez em relação ao conjugado de carga, 7th INDUSCON .

Pan, C.-T. and Sue, S.-M. (2005). A linear maximum torque per ampere control for ipmsm drives over full-speed range, IEEE Trans. on energy conversion

P\&D (2007). Motor de ímãs permanentes e inversor de frequência, WEG em Revista .

Pillay, P. and Krishnan, R. (1989). Modelling, simulation and analysis of permanent-magnet motor drives, part 1: The permanent-magnet synchronous motor drive, IEEE Trans. on industry applications

S. Laghrouche F., P., Glumineau, A. and Boisliveau, R. (2003). Robust second order sliding mode control for permanent magnet synchronous motor, American Control Conference ACC). .

Sozer, Y. and Torrey, D. A. (1998). Adaptative flux weakening control of permanent magnet synchronous motors, Thirty-Third IAS Annual Meeting .

Vas, P. (1998). Sensorless vector and direct torque control, Oxford University Press.

Øvrebø, S. (2004). Sensorless control of permanent magnet synchronous machines, $\mathrm{PhD}$ thesis, Norwegian University.

\section{APÊNDICE A - DADOS DO MOTOR}

Tabela 1: Dados do MSIPI

\begin{tabular}{c|c}
\hline \hline Parâmetro & Valor \\
\hline Potência nominal & $22 \mathrm{~kW}$ \\
Velocidade nominal & $3.800 \mathrm{rpm}$ \\
Velocidade máxima & $8.000 \mathrm{rpm}$ \\
Tensão nominal & $380 \mathrm{~V}$ \\
Corrente nominal & $40 \mathrm{~A}$ \\
Número de polos & 6 \\
Fcem & $0,085 \mathrm{~V} / \mathrm{rpm}$ \\
$\mathrm{J}$ & $0,06 \mathrm{~kg} \cdot \mathrm{m}^{2}$ \\
$r_{s}$ & $0,06 \Omega$ \\
$L_{d}$ & $1,00 \mathrm{mH}$ \\
$L_{q}$ & $2,00 \mathrm{mH}$ \\
\hline \hline
\end{tabular}

